\title{
[THU0352] BLINDED VS ULTRASOUND-GUIDED CORTICOSTEROID INJECTIONS FOR THE TREATMENT OF THE GREATER TROCHANTERIC PAIN SYNDROME (SDPT): A RANDOMIZED CONTROLLED TRIAL
}

\author{
G. Q. Estrela, R. Furtado, J. Natour, S. Narimatsu, A. Rosenfeld. Rheumatology Division, Universidade \\ Federal de Sao Paulo, Sao Paulo, Brazil
}

Background: The peritrochanteric corticosteroid injections with anesthesics is one of the suggested treatments for the refractory case of the Greater Throcanteric Pain Syndrome and this therapeutic intervention can be guided or not.

Objectives: To compare the effectiveness of both injections in the Greater Trochanteric Pain syndrome treatment: The blinded corticosteroid injections and the ultrasound-guided corticosteroid injections.

Methods: A prospective, randomised, double - blind, controlled study was made involving 60 patients (from 19 65 years old) who were diagnosed with GTPS for at least 30 days. The patients were randomised in two groups. Both groups were infiltraded once. Group $1(n=30)$ was infiltraded blindly. Group $2(n=30)$ was infiltrated with the ultrasound guidance. In both groups betamethasone $(6 \mathrm{mg})$ and lidocaine (2 cc) were used in a same syringe. Two blinded observers evaluated the experiment during week 0, 1, 4 and 8 after the procedure, using a visual analogue scale (VAS: 0-10). The patients were followed according: Spontaneous pain, painfull palpation, global health, external rotation pain, pain when testing against resistance, repositioning functional testing, "time up and go" (time in minutes), test six-minute walk (distance in meters), Lequesne hip; goniometry hip (flexion, extension and abduction), and ultrasound qualitative and quantitative (in $\mathrm{mm}$ ) measurements of the gluteus medium tendon, minimum tendon and trochanteric bursa. Side effects were observed and evaluated during the research.

Results: We studied 60 patients with a mean age of 54 years (10.1), mean duration of pain peritrochanteric of 32.2 months (35.8), with $98 \%$ of women, $90 \%$ white and $61.66 \%$ sedentary. After 8 weeks of follow-up, there was improvement over the initial time (intra-group evaluation) in both groups. Using repeated measures ANOVA, there was no significant difference between the groups for local pain VAS $(p=0.347)$, VAS of pain on palpation $(p=0.873)$, EVA external rotation $(p=0.215)$, test repositioning against resistance $(p=0.855)$, test "time up and go" $(p=0.062)$, test the six-minute walk $(p=0.537)$, Lequesne hip $(p=0.802)$. There was also no statistical difference in the sonographic assessment $(p>0.1)$. The variable VAS global health, was the only one that evolved differently between groups over time $(p=0.023)$ with better intergroup evaluation for group 2 . There were no significant adverse effects.

Conclusions: There are benefits when it comes to the patient's general perception, but according to this study, there is no benefit (intermediate-term) when ultrasound-guided infiltration is used in GTPS patients.

\section{References:}

Cohen SP, Strassels SA, Foster L, et al. Comparison of fluoroscopically guided and blind corticosteroid injections for greater trochanteric pain syndrome: multicentre randomized controlled trial. BMJ 2009; 338:b1088

Labrosse JM, Cardinal E, Leduc BE, et al. Effectiveness of ultrasound-guided corticosteroid injection for the treatment of gluteus medius tendinopathy. AJR 2010; 194:202-206

Shbeeb MI, Matteson EL. Trochanteric bursitis (greater trochanter pain syndrome). Mayo Clin Proc 1996; 71:565569

Ege Rasmussen KJ, Fanø N. Trochanteric bursitis: treatment by corticosteroid injection. Scand J Rheumatol 1985; $14: 417-420$

Acknowledgements: Ultrasound-guided; corticosteroid injections; Greater Trochanteric Pain Syndrome.

Disclosure of Interest: None declared

DOI: 10.1136/annrheumdis-2014-eular.5394

Citation: Ann Rheum Dis 2014;73(Suppl2): 304

Session: Back pain, mechanical musculoskeletal problems, local soft tissue disorders 